Purdue University

Purdue e-Pubs

ECE Technical Reports

Electrical and Computer Engineering

7-1-1992

\title{
Lower Bounds on Threshold and Related Circuits via Communication Complexity
}

\author{
V.P. ROYCHOWDHURY \\ Purdue University, School of Electrical Engineering \\ K.Y.SIU \\ Purdue University, School of Electrical Engineering \\ A. ORLITSKY \\ Purdue University, School of Electrical Engineering
}

Follow this and additional works at: http://docs.lib.purdue.edu/ecetr

ROYCHOWDHURY, V. P.; SIU, K. Y.; and ORLITSKY, A., "Lower Bounds on Threshold and Related Circuits via Communication Complexity" (1992). ECE Technical Reports. Paper 306.

http://docs.lib.purdue.edu/ecetr/306

This document has been made available through Purdue e-Pubs, a service of the Purdue University Libraries. Please contact epubs@purdue.edu for additional information. 


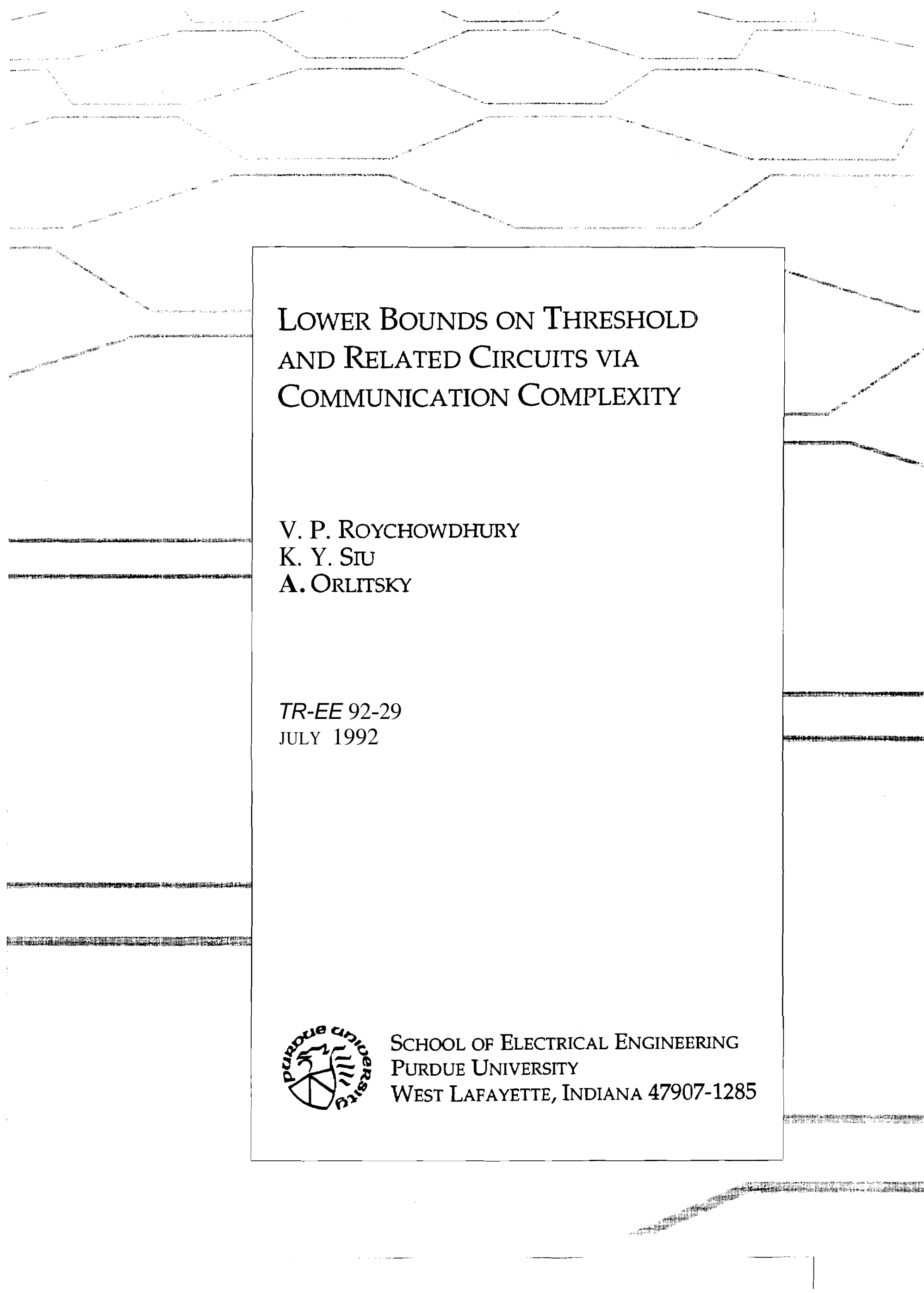




\title{
Lower Bounds on Threshold and Related Circuits via Communication Complexity
}

\author{
V. P. Roychowdhury * K. Y. Siu ${ }^{\dagger} \quad$ A. Orlitsky ${ }^{\ddagger}$
}

\begin{abstract}
Communication-complexity definitions and arguments are used to derive linear $(\Omega(n))$ and almost-linear $(\Omega(n / \log n))$ lower bounds on the size of circuits implementing certain functions. The techniques utilize only basic features of the gates used and of the functions implemented hence apply to a large class of gates (including unbounded fan-in AND/OR, threshold, symmetric, and generalized symmetric) and to a large class of functions (including equality, comparison, and inner product mod 2). Each of the bounds derived is shown to be tight for some functions and some applications to threshold-circuit complexity are indicated. The results generalize and in some cases strengthen results in $[1,2]$.
\end{abstract}

Index Terms: Linear/Almost-Linear Circuit-size Lower Bounds; Communication Complexity; Threshold gates/circuits; Symmetric gates/circuits; Equality, Comparison and Inner Product mod 2 Boolean functions.

'School of Electrical Eng., Purdue University, West Lafayette, IN 47907.

${ }^{\dagger}$ Department of Electrical and Computer Eng., University of California at Irvine, Irvine, CA 92717.

${ }^{\ddagger}$ AT\&T Bell Laboratories, 600 Mountain Avenue, Murray Hill, NJ 07974. 


\section{Introduction}

We describe the model, review known results, and introduce techniques and results presented in this paper.

\section{Gates, Circuits, and Complexity}

An $n$-variable Boolean function is a mapping

$$
f:\{0,1\}^{\{1, \ldots, n\}} \rightarrow\{0,1\}
$$

An element of $\{1, \ldots, n\}$ is a variable. An element of $\{0,1\}^{\{1, \ldots, n\}}$, viewed as a value assignment to the variables, is an input. If $\mathrm{x}$ is an input, then $f(x)$ is the corresponding output of $f$.

An n-variable gate is a physical device computing a single n-variable function. The input variables of a gate can be permuted, omitted, or repeated, hence we identify the gate with the set of functions derived by such operations. For example, the set of functions implementable by a gate computing the 4-variable function $(x \wedge y) \vee(z \wedge w)$, where $\wedge$ is logical "AND" and $\bigvee$ logical "OR," includes functions such as $(x \wedge z) \bigvee(y \wedge w),(x \wedge y) \bigvee(x \wedge y) \equiv x \wedge y$ and $(y \wedge y) \bigvee(y \wedge y) \equiv y$.

We usually consider a set, or a family, of gates. We identify the family with the union of the function sets corresponding to each of its gates.

Let $\mathcal{G}$ be a family of gates. A circuit whose gates are all from $\mathcal{G}$ is a $\mathcal{G}$-circuit. The size of a circuit is the number of gates it contains and its depth is the maximum number of gates along a path from an input to an output. The $\mathcal{G}$-circuit complexity $C_{\mathcal{G}}(f)$ of $\mathbf{f}$ is the size of the smallest $\mathcal{G}$-circuit that computes $\mathrm{f}$. In principle, some function may not be computed by a $\mathcal{G}$-circuit. However, every gate family considered here forms a complete basis, and hence $C_{\mathcal{G}}(\mathrm{f})$ is always defined.

The circuit complexity of functions has many theoretic and practical applications. Therefore, several gate families have been extensively investigated. They include:

$A N D / O R / N O T$ gates $(\mathcal{A O N}) \quad$ These gates perform logical "AND" or "OR" of their, possibly negated, inputs. AND/OR/NOT gates come in two varieties: constant fan-in gates and unbounded fan-in gates. The bounds we prove apply to both. 
Symmetric gates $(\mathcal{S Y \mathcal { M }})$ Gates of the form $g\left(\sum_{i=1}^{n} x_{i}\right)$ for arbitrary binary functions $\mathrm{g}$. These gates compute some binary function of their input sum.

One type of a symmetric gate is a mod, gate. It computes a binary function of the form $g\left(\left(\sum_{i=1}^{n} x_{i}\right) \bmod \mathrm{m}\right)$ for some constant integer $m$.

Threshold gates $(\mathcal{T} \mathcal{H}) \quad$ Gates of the form $\operatorname{sgn}\left(\sum_{i=1}^{n} w_{i} x_{i}-T\right)$ where $T$ is an arbitrary threshold, the $w_{i}$ 's are integer weights, and $\operatorname{sgn}(x)$ is 1 if $\mathrm{x} \geq 0$ and 0 otherwise.

In the analysis we distinguish between general (arbitrary weight) threshold gates and polynomial-weight threshold gates where the $w_{i}$ s are restricted to be polynomial in $n$.

Generalized symmetric gates $(\mathcal{G S})$ Gates of the form $g\left(\sum_{i=1}^{n} w_{i} x_{i}\right)$ for arbitrary function $\mathrm{g}$ and weights $w_{i}$ that are polynomial in $\mathrm{n}$.

The weights are restricted to be polynomial because every function can be computed by a single generalized symmetric gate with arbitrary weights.

Note that every AND/OR/NOT gate is also a polynomial-weight threshold gate and that any polynomial-weight threshold gate as well as any symmetric gate is also a generalizedsymmetric gate.

\section{Related Results and Motivation}

Much research has gone into estimating $C_{\mathcal{G}}(f)$ for various functions and gate families [3]. The strongest results apply to bounded-depth circuits. For constant depth AND/OR/NOT circuits and mod, circuits (where $\mathrm{p}$ is prime), $[4,5,6]$ established exponential-size lower bounds for specific functions such as the parity. For more powerful circuits, less is known. For example, [7] proved an exponential-size lower bound on the size of depth-2 threshold circuits implementing the n-variable inner product mod 2 function:

$$
\operatorname{IP}\left(x_{1}, \ldots, x_{\frac{n}{2}}, y_{1}, \ldots, y_{\frac{n}{2}}\right)= \begin{cases}1 & \text { if } \sum_{i=1}^{\frac{n}{2}} x_{i} \wedge y_{i} \\ 0 & \text { otherwise }\end{cases}
$$

However, this bound applies only when the weights in the second layer are restricted to be polynomial. No superlinear lower bounds are known for depth-':! threshold circuits with exponential weights in the second layer, or for depth-3 threshold circuits with polynomial weights. 
For unrestricted-depth unbounded-fan-in circuits even weak lower bounds, such as linear or logarithmic in the number of input variables, are considered difficult to prove [3, 1]. For example, an $\Omega(\log n)$ lower bound on the size of threshold circuits computing the parity of $n$ bits is shown in [3]. Only recently have linear/almost-linear lower bounds been established for circuits with gates of unbounded fan-in. A linear-size lower bound on circuits where each gate computes a commutative and associative function, was given in [8]. However, the family of gates is too restrictive to apply to symmetric or threshold circuits.

Recently, [1] established an $\Omega(n / \log n)$ lower bound on the size of symmetric-gate circuits computing the n-variable equality function:

$$
\operatorname{EQ}\left(x_{1}, \ldots, x_{\frac{n}{2}}, y_{1}, \ldots, y_{\frac{n}{2}}\right)= \begin{cases}1 & \text { if } x_{i}=y_{i} \text { for all } 1 \leq i \leq \frac{n}{2} \\ 0 & \text { otherwise }\end{cases}
$$

Novel techniques such as analytic-function interpolation of Boolean functions and the differential dimension were used. More recently [2] proved a linear lower bound $(n / 4)$ on the size of arbitrary-weight threshold circuits computing the n-variable IP .

\section{Techniques and Results in this Paper}

Using communication-complexity concepts and techniques, we derive linear and almost-linear lower bounds on the size of circuits implementing certain functions. This approach utilizes only basic features of the gates used, hence the bounds hold for general families of gates of which the symmetric and threshold gates considered in $[1,2]$ are special cases. Thus communication complexity arguments serve to generalize known lower bounds and unify their proofs.

In the next section we define the decomposition number and the largest monochromatic rectangle of a function. These are simple attributes that have proven useful in analyzing the communication complexity of various functions.

In Section 3 we consider polynomially-rectangular gates. These gates, which include symmetric, generalized symmetric, and polynomial-weight threshold gates, compute functions with small decomposition numbers. We show that functions computed by small-size circuits of polynomially-rectangular gates have small decomposition numbers. It follows that functions with high decomposition numbers require large circuits. We then use some effective techniques that have been developed to lower bound decomposition numbers to prove almost-linear lower bounds on the circuit complexity of several functions. 
In Section 4 we strengthen the results for triangular gates. These gates, which include all threshold gates, compute functions with large monochromatic rectangles. We show that any function computed by a small circuit of triangular gates contains a large monochromatic rectangle. Therefore, functions with only small monochromatic rectangles require large, in some cases linear-size, circuits.

We illustrate the results using the equality and the inner product mod 2 functions defined earlier in this section. The bounds we derive imply:

1. Any implementation of $n$-variable EQ or IP by generalized symmetric gates requires about $n / \log \mathrm{n}$ gates. Namely, if the weights are bounded by $\mathrm{n}^{\mathrm{k}}$, then

$$
\frac{1}{4(\mathrm{k}+1) \log \mathrm{n}} \leq C_{\mathcal{G S}}(\mathrm{EQ}), C_{\mathcal{G S}}(\mathrm{IP}) \leq \frac{\log 3}{2 k} \frac{\mathrm{n}}{\log n}
$$

2. Any implementation of $n$-variable EQ or IP by symmetric gates requires at least $\frac{n}{\log n}$ gates:

$$
C_{\mathcal{S Y M}}(\mathrm{EQ}), C_{\mathcal{S Y M}}(\mathrm{IP}) \geq \frac{n}{4 \log n} .
$$

3. Any implementation of $n$-variable EQ or IP by AND/OR/NOT gates requires about n gates:

$$
\frac{\mathrm{n}}{2 \log 3} \leq C_{\mathcal{A O N}}(\mathrm{EQ}), C_{\mathcal{A O N}}(\mathrm{IP}) \leq 2 n
$$

4. Any implementation of $n$-variable IP by threshold gates requires about $n$ gates.

$$
\frac{1}{4} n \leq C_{\tau \mathcal{H}}(\mathrm{IP}) \leq \frac{3}{4} n+1
$$

Both upper and lower bounds apply to arbitrary- and polynomial-.weight threshold circuits.

Note that the bounds in (1), (2), and (3) are tight up to a small multiplicative factor.

Related to EQ is the n-variable comparison function:

$$
\operatorname{COMP}\left(x_{1}, \ldots, x_{\frac{n}{2}}, y_{1}, \ldots, y_{\frac{n}{2}}\right)= \begin{cases}1 & \text { if } \sum_{i=1}^{\frac{n}{2}} 2^{i} x_{i} \geq \sum_{i=1}^{\frac{n}{2}} 2^{i} y_{i} \\ 0 & \text { otherwise }\end{cases}
$$

Although we do not discuss COMP explicitly, it shares the same size bounds as EQ . 


\section{Communication Complexity Arguments}

As before, let $\mathrm{f}:\{0,1\}^{\{1, \ldots, n\}} \rightarrow\{0,1\}$ be an $\mathrm{n}$-variable Boolean function. Recall that an element of $\{1, \ldots, n\}$ is a variable and an element of $\{0,1\}^{\{1, \ldots, n\}}$ is an input. If $X$ is a set of variables then an element of $\{0,1\}^{X}$ is a value assignment to the variables in $\mathrm{X}$ and is called an $\mathrm{X}$-input.

Let $\{\mathrm{X}, \mathrm{Y})$ partition the set of variables $(X \cup Y=\{1, \ldots, n\}$ and $X \cup Y=0)$. An X-input $\mathrm{X}$ together with a $\mathrm{Y}$-input y correspond in an obvious way to an input which we call the joint input and denote by $(x, y)$. In the same way, the set of all inputs corresponds to the Cartesian product $\{0,1\}^{X} \times\{0,1\}^{Y}$. We can therefore associate with the function $\mathrm{f}$ and the partition $\{\mathrm{X}, \mathrm{Y})$ a matrix $M_{f, X, Y}$. It has $2^{|X|}$ rows, each indexed by an X-input, $2^{|Y|}$ columns, each indexed by a $\mathrm{Y}$-input, and

$$
M_{f, X, Y}(x, y)=f(x, y)
$$

An $\{\mathrm{X} ., Y\}$-rectangle is a Cartesian product $\mathbf{A} \times \mathbf{B}$ where $\mathbf{A}$ is a set of $\mathrm{X}$-inputs and $\mathrm{B}$ is a set of $\mathrm{Y}$-inputs. The sire of the rectangle is $|A| \cdot|B|$, the number of inputs it contains. An $\{X, Y\}$-decomposition is a partition of $\{0,1\}^{X} \times\{0,1\}^{Y}$ into $\{X, Y\}$-rectangles. The sire of the decomposition is the number of rectangles in the partition. A set of inputs is f-constant if $\mathrm{f}$ assigns the same value to all its elements. An f-constant $\{\mathrm{X}, Y\}$-decomposition is an $\{\mathrm{X}, Y\}$-decomposition whose rectangles are all $\mathrm{f}$-constant.

Rectangles play a major role in the following communication complexity problem. As before, let $\mathrm{f}$ be an $\mathrm{n}$-variable Boolean function and $\{\mathrm{X}, \mathrm{Y})$ a partition of the variables. A person $P_{\mathcal{X}}$ knows an $\mathrm{X}$-input, a person $P_{\mathcal{Y}}$ knows a $\mathrm{Y}$-input, and they communicate according to a predetermined protocol in order to find the value of $f$ on their joint input. We are interested in $\hat{C}(f, \mathrm{X}, \mathrm{Y})$, the number of bits $P_{\mathcal{X}}$ and $P_{\mathcal{Y}}$ must transmit for the worst input.

As shown by [9],

1. Every protocol induces an $\{\mathrm{X}, Y\}$-decomposition.

2. If the protocol always produces the correct answer, this decomposition is f-constant.

3. The number of bits required by the protocol for the worst input is at least the logarithm ${ }^{1}$

'All logarithms are to the base 2 . 
of the size of the decomposition.

Let $\rho_{f, X, Y}$ be the smallest size of an f-constant $\{\mathrm{X}, Y\}$-decomposition. From the above,

$$
\hat{C}(f, X, Y) \geq \log \rho_{f, X, Y} .
$$

Aho, Ullman, and Yanakakis [10] showed that this bound is not far from being tight:

$$
\hat{C}(f, X, Y) \leq \log ^{2} \rho_{f, X, Y}
$$

For that reason, several simple methods were introduced to lower bound $\rho_{f, X, Y}$ for arbitrary $\mathbf{f}, \mathrm{X}$, and $\mathrm{Y}$.

\section{Largest $f$-constant rectangle}

Let $\mathrm{L}_{f, X, Y}$ be the size of the largest $\mathrm{f}$-constant $\{\mathrm{X}, Y\}$-rectangle. Clearly,

$$
\rho_{f, X, Y} \geq \frac{2^{n}}{L_{f, X, Y}}
$$

\section{Fooling set}

An $f$-constant subset $S$ of $\{0,1\}^{X} \mathrm{x}\{0,1\}^{Y}$ is an $\{\mathrm{X}, Y\}$-fooling set if $\left(x_{1}, y_{1}\right),\left(x_{2}, y_{2}\right) \in$ $\mathrm{S}$ implies that either $\mathrm{f}\left(x_{1}, y_{2}\right)$ or $\mathrm{f}\left(x_{2}, y_{1}\right)$ differs from the common value of $\mathrm{f}$ over $\mathrm{S}$. Let $F_{f, X, Y}$ be the size of the largest $\{\mathrm{X}, Y\}$-fooling set. An f-constant $\{\mathrm{X}, Y\}$-rectangle contains at most one element of a given $\{X, Y\}$-fooling set, hence:

$$
\rho_{f, X, Y} \geq F_{f, X, Y}
$$

\section{Rank}

The matrix representing the indicator function of a rectangle has rank 1, and ranks are subadditive under matrix addition. Melhorn and Schmidt [11] concluded that under any field

$$
\rho_{f, X, Y} \geq \operatorname{rank}\left(M_{f, X, Y}\right)
$$

In our applications, we can choose the most advantageous partition of the input variables. We therefore define the decomposition number of $\mathbf{f}$,

$$
\rho_{f} \stackrel{\text { def }}{=} \max \left\{\rho_{f, X, Y}:\{X, Y) \text { partitions }\{1, \ldots, n\}\right\}
$$

to be the number of rectangles needed in the variable partition that yields the strongest bound in (1). We use the methods above to lower bound the decomposition number of our two functions. 
Example 1 We show that the decomposition numbers of both EQ and IP are larger than $2^{\frac{n}{2}}$. In the following, $X=\left\{1, \ldots, \frac{n}{2}\right\}$ and $Y=\left\{\frac{n}{2}+1, \ldots, n\right\}$. Every $\frac{n}{2}$-bit sequence corresponds in an obvious way to an $\mathrm{X}$-input and to a $\mathrm{Y}$-input. We can therefore talk about the joint input $(x, x)$ where $x \in\{0,1\}^{\frac{n}{2}}$.

Equality The set $\{(\mathrm{x}, \mathrm{x}): \mathrm{x} \in\{0,1\} \mathrm{f}\}$ is an $\{\mathrm{X}, Y\}$-fooling set of size $2^{\frac{\mathrm{n}}{2}}$, implying that $\rho_{\mathrm{EQ}, X, Y} \geq 2 \mathrm{f}$. In fact, $\rho_{e q}=\rho_{\mathrm{EQ}, X, Y}=2^{\frac{n}{2}+1}$.

Inner product $\bmod 2 \quad M_{\mathrm{IP}, X, Y}$ has full rank over the reals, hence $\rho_{\mathrm{IP}} \geq 2^{\frac{n}{2}}$.

\section{Rectangular gates}

The last section was motivated by the notion that a function with a high decomposition number is "complicated." To show that computing such a function requires many gates, we now show that the gates used are "simple," that is, they can be decomposed into a small number of' rectangles.

A function $\mathrm{f}$ is $\mathrm{r}$-rectangular for some integer $r$ if for every variable partition $\{\mathrm{X}, \mathrm{Y}$ ) there is an $\mathrm{f}$-constant $\{\mathrm{X}, Y\}$-decomposition consisting of at most $r$ rectangles. Namely, if

$$
\rho_{f} \leq r
$$

Let $\mathrm{p}: \mathcal{Z}^{+} \rightarrow 2$. A family $\mathcal{G}$ of functions is $\mathrm{p}$-rectangular if for every $\mathrm{m} \leq \mathrm{n}$, all $\mathrm{m}$ variable functions in $\mathcal{G}$ are $p(n)$-rectangular. The family is polynomially-rectangular if it is prectangular for some polynomial $\mathrm{p}$. These definition apply to gates and families of gates via their underlying functions. The next lemma, its simple proof omitted, provides a basic tool for proving that a function is $r$-rectangular.

Le mma 1. Let $\mathbf{f}$ be a Boolean function and let $\{\mathrm{X}, \mathrm{Y})$ partition the set of variables. If $\mathrm{f}(\mathrm{x}, \mathrm{y})$ can be expressed as $h\left(g_{1}(x), g_{2}(y)\right)$ then

$$
\rho_{f, X, Y} \leq\left|g_{1}\right| \cdot\left|g_{2}\right|
$$

where $\left|g_{i}\right|$ is the size of the range of $g_{i}$.

To prove that a function is $r$-rectangular we apply the lemma to all possible partitions of the variables. 
Example 2 We show that the gate families mentioned in the introduction are polynomially rectangular. In the following, $\{\mathrm{X}, \mathrm{Y})$ is an arbitrary partition of $\{1, \ldots, n\}$.

\section{AND/OR/NOT gates}

$$
\bigwedge_{i \in\{1, \ldots, n\}} x_{i}=\left(\bigwedge_{i \in X} x_{i}\right) \bigwedge\left(\bigwedge_{i \in Y} x_{i}\right)
$$

hence the lemma implies that every AND gate is 4-rectangular (three rectangles suffice). The same holds for NOT gates.

\section{Symmetric gates}

$$
f(x, y)=h\left(\sum_{i \in X} x_{i}, \sum_{i \in Y} y_{i}\right),
$$

hence

$$
\rho_{f, X, Y} \leq(|X|+1) \cdot(|Y|+1) \leq\left(\frac{n}{2}+2\right)^{2}
$$

\section{Generalized symmetric gates}

$$
\mathrm{f}(x, y)=h\left(\left(\mathrm{C}_{i \in X} w_{i} x_{i}+\sum_{i \in Y} w_{i} x_{i}\right) .\right.
$$

where the $w_{i}$ 's are bounded by some polynomial $p(n)$. The first sum attains at most $(|\mathrm{X}|+1) \cdot p(n)$ values and likewise for the second, hence $\mathrm{f}$ is $\left(\frac{n}{2}+1\right)^{2} \cdot p^{2}(n)$-rectangular. It follows that the family of generalized symmetric functions (and in particular, of polynomial-weight threshold circuits) is polynomially rectangular.

Lemma 2 Let $\mathcal{G}$ be a $p$-rectangular family of gates. If an $\mathcal{G}$-circuit consisting of k gates computes an n-variable function $f$, then

$$
\rho_{f} \leq(p(n))^{k}
$$

Proof: Order the gates in the circuit so that if $i<\mathbf{j}$ then gate $i$ does not follow gate $\mathbf{j}$. Let $g_{j}$ denote the function computed by gate $\mathbf{j}$. We prove by induction on $\mathbf{j}$ that the vector-valued function $G_{j} \stackrel{\text { def }}{=}\left(g_{1}, g_{2}, \ldots, g_{j}\right)$ has $\rho_{G}, X, Y \leq(p(n))^{j}$ for all variable partitions $\{X, Y)$. The lemma will follow.

The induction basis holds by definition; suppose it holds for $\mathbf{j}$, and consider the $(\mathbf{j}+1)$ st gate. Let $\{\mathrm{X}, \mathrm{Y})$ be a variable partition. There is a $G_{j}$-constant $\{\mathrm{X}, \mathrm{Y}\}$-decomposition 
consisting of at most $(p(n))^{j}$ rectangles. Let $\mathrm{R}$ be a rectangle in this decomposition. Over $\mathrm{R}$, all of $g_{1}, \ldots, g_{j}$ are constant, hence the $(\mathrm{j}+1)$ st gate coincides with a $p(n)$ rectangular function of the original variables. Therefore $\mathrm{R}$ can be partitioned into $p(n) G_{j+1}$-constant $\{\mathrm{X}, Y\}$ rectangles, and the induction step follows.

Corollary 1 Let $\mathcal{G}$ be a p-rectangular family of gates. For every n-variable function $\mathrm{f}$,

$$
C_{\mathcal{G}}(f) \geq \frac{\log \rho_{f}}{\log p(n)}
$$

We apply the corollary to lower bound the number of gates needed to implement our two functions.

\section{Corollary 2}

1. For circuits consisting of AND, OR, and NOT gates:

$$
\frac{n}{2 \log 3} \leq C_{\mathcal{A O N}}(\mathrm{EQ}), C_{\mathcal{A O N}}(\mathrm{IP}) \leq 2 n
$$

2. For circuits consisting of-generalized symmetric gates:

$$
C_{\mathcal{G S}}(\mathrm{EQ}), C_{\mathcal{G S}}(\mathrm{IP}) \in \Theta\left(\frac{n}{\log n}\right)
$$

More specifically, if the weights are bounded by $\mathrm{n}^{\mathrm{k}}$, then

$$
\frac{1}{4(\mathrm{k}+1) \log \mathrm{n}} \leq C_{\mathcal{G S}}(\mathrm{EQ}), C_{\mathcal{G S}}(\mathrm{IP}) \leq \frac{\log 3}{2 k} \frac{\mathrm{n}}{\log n} .
$$

3. For circuits consisting of symmetric gates:

$$
C_{\mathcal{S Y M}}(\mathrm{EQ}), C_{\mathcal{S Y M}}(\mathrm{IP}) \geq \frac{n}{4 \log n} .
$$

Proof: All six lower bounds follow from Corollary 1 as both EQ and IP have decomposition numbers of at least $2^{\frac{n}{2}}$. The upper bounds in (1) follow from a simple construction. To prove the upper bounds in (2) we implement EQ as a depth-2 threshold circuit, yielding a simple circuit with slightly more gates than the upper bound. We implement IP as a depth-3 generalized symmetric circuit (the next section shows it cannot be impleinented using less than $\mathrm{n}$ threshold gates). 
Let $\mathrm{m}=2\lfloor k \log n\rfloor$. Clearly, m-variable COMP can be written as

$$
\operatorname{COMP}\left(x_{1}, \ldots, x_{\frac{m}{2}}, y_{1}, \ldots, y_{\frac{m}{2}}\right)=\operatorname{sgn}\left(\sum_{i=1}^{m / 2} 2^{i}\left(x_{i}-y_{i}\right)\right),
$$

thus can he implemented by a single threshold gate with weights of at rnost $\mathrm{n}^{\mathrm{k}}$. For $i=$ $1, \ldots,\lceil n / m\rceil$, let $\mathrm{x}^{\mathrm{i}}=x_{(i-1) \cdot m / 2+1}, \ldots, x_{i \cdot m / 2}$ and $y^{i}=y_{(i-1) \cdot m / 2+1}, \ldots, y_{i \cdot m / 2}$. Then,

$$
\operatorname{EQ}\left(x^{i}, y^{i}\right)=\operatorname{COMP}\left(x^{i}, y^{i}\right)+\operatorname{COMP}\left(y^{i}, \mathrm{x}^{\mathrm{i}}\right)-1 \text {. }
$$

Hence, m-variable EQ can be implemented by a depth-2 threshold circuit with weights of at most $n^{k}$ and where the top gate is just a weighted sum of the first-level outputs (without a threshold). Finally, observe that

$$
\operatorname{EQ}\left(x_{1}, \ldots, x_{\frac{n}{2}}, y_{1}, \ldots, y_{\frac{n}{2}}\right)=\bigwedge_{i=1}^{[n / m]} \operatorname{EQ}\left(x^{i}, y^{i}\right)
$$

Since any AND is just the sum of its variables with an appropriate threshold, this gate can be combined with the second layer above to derive a depth-2 circuit for EQ of size $2\lceil n / 2 k \log n\rceil+1$. When generalized symmetric gates are used instead of threshold gates, the number of gates can be reduced to $\lceil n / 2 k \log n\rceil+1$.

When trying to meet the lower bound for IP, we cannot use threshold gates as we did for EQ. The next section shows that any threshold circuit for IP (even with exponential weights) has at least linear size. Yet, we can use the circuit structure applied to EQ. Every $(\mathrm{k} \log n)$ variable function, in particular $\operatorname{IP}\left(x_{1}, \ldots, x_{k \log n / 2}, y_{1}, \ldots, y_{k \log n / 2}\right)$, can be computed by a single generalized symmetric gate with weights of at most $\mathrm{n}^{\mathrm{k}}$. Use $\lceil n / k \log n\rceil$ generalized symmetric gates to compute the partial IP's, then use a single (symmetric) gate to compute their parity.

A note on COMP : Equation (2) shows that n-variable COMP can be computed by a single threshold gate with exponential weights. However, if the weights are polynomially bounded, then as noted in the introduction, the lower bound on EQ can be modified to show that $C_{\mathcal{G S}}(\mathrm{COMP}) \geq \Omega(n / \log \mathrm{n})$. Thus a single threshold gate with polynomial weights cannot compute COMP. We next show that the lower bound can be met by a depth-3 polynomial-weight threshold circuit. It is not known whether the lower bound can be met by a polynomial-weight threshold circuit of depth two. 
Let $\mathrm{m}=2\lceil\log n\rceil$. For $i=1, \ldots,\lceil n / m\rceil$, let

$$
C_{i}=\operatorname{sgn}\left(\sum_{j=(i-1) \cdot m / 2+1}^{i \cdot m / 2} 2^{j}\left(x_{j}-y_{j}\right)\right),
$$

and

$$
\tilde{C}_{i}=\operatorname{sgn}\left(\sum_{j=(i-1) \cdot m / 2+1}^{i \cdot m / 2} 2^{j}\left(x_{j}-y_{j}\right)-1\right) .
$$

Note that both $C_{\boldsymbol{i}}$ and $\tilde{C}_{\boldsymbol{i}}$ can be computed with threshold gates of polynomially bounded weights. Further,

$$
C_{i}=1 \text { iff } \sum_{j=(i-1) \cdot m / 2+1}^{i \cdot m / 2} 2^{j} x_{j} \geq \sum_{j=(i-1) \cdot m / 2+1}^{i \cdot m / 2} 2^{j} y_{j}
$$

and

$$
\tilde{C}_{i}=1 \text { iff } \sum_{j=(i-1) \cdot m / 2+1}^{i \cdot m / 2} 2^{j} x_{j}>\sum_{j=(i-1) \cdot m / 2+1}^{i \cdot m / 2} 2^{j} y_{j}
$$

Define Boolean expressions

$$
\begin{gathered}
B_{\lceil n / m\rceil}=\tilde{C}_{\lceil n / m\rceil} \\
B_{k}=\tilde{C}_{k} \bigwedge_{j=k+1}^{\lceil n / m\rceil} C_{j} \quad \text { for } k=2, \ldots,\lceil n / m\rceil-1 \\
\text { and } \quad B_{1}=\bigwedge_{j=1}^{\lceil n / m\rceil} C_{j}
\end{gathered}
$$

It is straightforward to see that

$$
\operatorname{COMP}\left(x_{1}, \ldots, x_{n}, y_{1}, \ldots, y_{n}\right)=\bigvee_{j=1}^{n} B_{j}
$$

The first layer of our circuit for the COMP function has $O(n / \log n)$ gates computing the $C_{i}$ and $\tilde{C}_{i}$. With these computed values as inputs, the second layer has $O(n / \log n)$ gates each computing the $B_{j}$. Finally the output gate computes the OR (V) of all the $B_{j}$ 's. The total number of gates is $O(n / \log n)$.

\section{Triangular Gates}

$\boldsymbol{A}$ matrix iis strictly triangular if all its rows and columns are nondecreasing. In a strictly triangular Boolean matrix, the sets of 1's and 0's resemble a (possibly truncated) triangle, 
hence the name. A matrix is triangular if its rows and columns can be permuted so that the resulting matrix is strictly triangular.

Lemma 3 (Alternative Definition) A binary matrix is triangular if and only if it contains no 2 by 2 rectangle of the form

$\begin{array}{lllll}0 & 1 & & 1 & 0 \\ 1 & 0 & \text { or } & 0 & 1\end{array}$

(recall that a rectangle need not be contiguous).

Proof: Row and column permutations preserve this non-containment property, so "only if $^{\mathrm{n}}$ is clear. For the other direction, permute the rows so that the number of 1's in each row is non decreasing, then permute the columns so that the number of 1 's in each column is non decreasing. The resulting matrix is strictly triangular for if in some column a 1 appears above a 0 , then, as the numbers of $1^{7}$ s does not decrease with the rows, there must be another column where in the same locations a 0 appears above a 1 , contradicting the non-containment assumption.

Some :properties of triangular matrices are apparent:

1. Every submatrix of a triangular matrix is triangular.

Proof: Obvious by either definition.

2. Every triangular matrix contains a constant rectangle of $1 / 4$ the size.

Proof: Permute the rows/columns till you get a strictly-triangular matrix. Consider the mid point $(\mathrm{x}, \mathrm{y})$. If the $(\mathrm{x}, y)$ th element of the matrix is 0 then the rectangle above and to the left of $(\mathrm{x}, \mathrm{y})$ is all 0 , otherwise, the rectangle to the right and below $(\mathrm{x}, \mathrm{y})$ is all 1.

3. Every submatrix of a triangular matrix contains a constant rectangle of $1 / 4$ its size.

Proof: Combine properties 1 and 2.

An n-variable function $\mathrm{f}$ is triangular if $M_{f, X, Y}$ is triangular for all $\{X, \mathrm{Y})$-partitions of the variables. A family of functions is triangular if all the functions in the family are. The definition applies to gates and families of gates via the underlying functions. 
Example 3 ([2]) Threshold gates (and in particular AND and OR gates) are triangular. We use the Lemma 3. Let $\mathrm{f}(\mathrm{x}, \mathrm{y})=\operatorname{sgn}\left(\sum u_{i} x_{i}+\sum v_{i} y_{i}\right)$. Suppose that $\mathrm{f}(\mathrm{x}, \mathrm{y})=\mathrm{f}\left(x^{\prime}, y^{\prime}\right)=1$ and that $\mathrm{f}\left(x, y^{\prime}\right)=\mathrm{f}\left(\mathrm{x}^{\mathrm{t}}, \mathrm{y}\right)=0$. Then $\sum u_{i} x_{i}+\sum v_{i} y_{i}>\sum u_{i} x_{i}+\sum v_{i} y_{i}^{\prime}$ while $\sum u_{i} x_{i}^{\prime}+$ $\sum v_{i} y_{i}<\sum u_{i} x_{i}^{\prime}+\sum v_{i} y_{i}^{\prime}$. Impossible.

Recall that $\mathrm{L}_{f, X, Y}$ was defined to be the size of the largest f-constant $\{\mathrm{X}, Y\}$-rectangle. Define $\mathrm{L}_{f}$ to be $\mathrm{L}_{\boldsymbol{f}, X, Y}$ for the most advantageous partition of the variables:

$$
L_{f} \stackrel{\text { def }}{=} \min \left\{L_{f, X, Y}:\{X, Y\} \text { partitions }\{1, \ldots, n\}\right\}
$$

Lemma 4 If a circuit consisting of $k$ triangular gates computes a function $f$ then

$$
L_{f} \geq \frac{2^{n}}{4^{k}}
$$

Proof: As in Lemma 2, order the gates in the circuit so that if $\mathbf{i}<\mathbf{j}$ then gate $\mathrm{i}$ does not follow. gate $\mathbf{j}$. Let $g_{j}$ denote the function computed by gate $\mathbf{j}$. We prove by induction on $\mathbf{j}$ that the vector-valued function $G_{j} \stackrel{\text { def }}{=}\left(g_{1}, g_{2}, \ldots, g_{j}\right)$ has $L_{G_{j}, X, Y} \geq \frac{2^{n}}{4^{k}}$ for all variable partitions $\{X, Y)$. The lemma will follow.

The induction basis holds by property (2) above. Suppose it holds for $\mathrm{j}$, and consider the $(j+1)$ st gate. Let $\{X, Y)$ be a variable partition. By induction hypothesis, there is a $G_{j}$-constant $\{\mathrm{X}, Y\}$-rectangle $\mathrm{R}$ of size $2^{n} / 4^{k}$. Over $\mathrm{R}$, the outputs of the first $\mathbf{j}$ gates are fixed, hence the input to the $(j+1)$ st gate varies only with the original inputs. It follows that over $\mathrm{R}$ the $(j+1)$ st gate coincides with a triangular function whose inputs are the original inputs. By property (3), there must be a subrectangle of $\mathrm{R}$ of size $\geq|R| / 4$ over which the $(j+1)$ st gate has a constant output.

Corollary 3 For every function $\mathbf{f}$ and every family $\mathcal{G}$ of triangular gates,

$$
C_{\mathcal{G}}(f) \geq \frac{\mathrm{n}-\log L_{f}}{2}
$$

Example 4 Let $X=\left(1, \ldots, \frac{n}{2}\right\}$ and $Y=\left\{\frac{n}{2}+1, \ldots, n\right\}$. Lindsey [12] showed that the largest IP-constant $\{X, Y\}$-rectangles are of size at most $2^{\frac{n}{2}}$. Hence

$$
C_{\text {TM }}(\text { IP }) \geq \frac{n}{4} .
$$


The bound on $C_{T \mathcal{H}}(\mathrm{IP})$ is asymptotically tight too. A simple depth-3 circuit computes IP using $\frac{3}{4} n+1$ polynomial-weight threshold gates. In a sense, this circuit is depth optimal too. [7] showed that every depth-2 threshold circuit for IP has exponential size if the weights at the seconcl layer are polynomial. It is not known whether there is a polynomial size depth-2 threshold circuit for IP when exponential weights are allowed at the second layer.

\section{Application To Threshold Circuits}

We briefly discuss some applications of the results and techniques discussed in the previous sections to threshold-circuit complexity.

Depth-Weight Tradeoffs in Threshold Circuits

Recent results [13] have shown that any depth-d threshold circuit (with arbitrary weights) can be simulated by a depth-(d+1) polynomial-weight threshold circuit with only a polynomial factor increase in size (for fixed d). However, no upper- or lower-bounds have been shown for the degree of this polynomial.

One can implement the n-variable EQ using only 3 threshold gates in depth-2. Yet Corollary 2 gave a lower bound of $\Omega(n / \log n)$ on the size of any polynomial-weight threshold circuits for EQ. We therefore have:

Corollary 4 There are n-variable functions whose polynomial-weight threshold-circuit complexity (regardless of depth) is at least $n / \log n$ times larger than their unrestrictedweight depth-3 threshold-circuit complexity.

Weighted-Sum gates

In our discussions, we often observed that the output gate of a given threshold circuit does not always require the sgn function usually associated with a threshold gate. A gate that computes a linear combination $\sum w_{i} x_{i}$ of its inputs (without taking a threshold) is a weighted-sum gate. No explicit function is known that requires super-polynomial size when implemented by a depth-2 arbitrary-weight threshold circuit with a weighted-sum gate at the output. This is a special case of the more difficult open problem of proving that some given function requires super-polynomial size when implemented by a depth-:! arbitrary-weight threshold circuit (with a threshold allowed in the output gate). We prove a partial result regarding weighted-sum gates in the context of the equality and other related functions. 
As mentioned earlier, the n-variable EQ can be implemented by a depth-2 circuit consisting of 2 threshold gates with exponential weights in the first layer and a weighted-sum gate in the second layer. We show that any circuit for EQ that consists of polynomial-weight threshold gates at the first layer and of a weighted-sum gate at the second layer (possibly with exponential weights) has exponential size.

Lemma 5 Suppose that a depth-2 circuit consisting of $p(n)$-rectangular gates in the first layer and a weighted-sum gate (possibly with exponential weights) at the output computes the $n$-variable EQ. Then the size of the circuit is at least $2^{\frac{n}{2}} / p(n)$.

Proof: Let $g_{1}, \ldots, g_{k}$ be the output functions of the $\mathrm{k}$ gates in the first layer of the circuit. Consider the 'natural' partition $X=\left\{1, \ldots, \frac{n}{2}\right\}$ and $Y=\left\{\frac{n}{2}+1, \ldots, n\right)$ of the input variables. Since the output function is a weighted sum of $g_{i}$ 's we have

$$
M_{\mathrm{EQ}, X, Y}=\sum_{i=1}^{k} w_{i} M_{g_{i}, X, Y}
$$

By subadditivity of ranks,

$$
\operatorname{rank}\left(M_{\mathrm{EQ}, X, Y}\right) \leq \sum_{i=1}^{k} \operatorname{rank}\left(M_{g_{i}, X, Y}\right)
$$

But

$$
\operatorname{rank}\left(M_{\mathrm{EQ}, X, Y}\right)=2^{\frac{n}{2}}
$$

and for all. $i \in\{1, \ldots, k\}$,

$$
p(n) \geq \rho_{g_{i}, X, Y} \geq \operatorname{rank}\left(M_{g_{i}, X, Y}\right)
$$

The lemma follows.

Corollary 5 Suppose that a depth-2 circuit consisting of polynomial-weight threshold gates in the first layer and a weighted-sum gate (possibly with exponential weights) at the output, computes the n-variable EQ. Then the size of the circuit is $\Omega\left(2^{\frac{n}{2}-\epsilon}\right)$ for every $\epsilon>0$. Proof: Example 2 implies that any threshold gate with weights bounded by $p(n)$ is $\left(\frac{\pi}{2}+\right.$ $1)^{2} p^{2}(n)$ rectangular.

The above result holds for all functions $f$, (e.g., COMP) for which $\operatorname{rank}\left(M_{f, X, Y}\right)$ is exponentially large for some partition $\{\mathrm{X}, \mathrm{Y}\}$ of the input variables. 


\section{Concluding Remarks}

Several problems remain unresolved.

1. The best symmetric-gates lower bounds for EQ and IP are $\Omega(n / \log n)$ while the best upper bounds are linear.

2. Is there a two-layer polynomial-weight threshold circuit for COMP that meets the lower bound of $\Omega(n / \log n)$ ?

3. The set of polynomially-rectangular gates, introduced in Section 3, includes the set of generalized symmetric gates. Are the two sets the same? Similarly the set of triangular gates, introduced in Section 4, includes the set of threshold gates. Are these sets the same? 


\section{APPENDIX}

\section{A lower bound on the Differential Dimension of Boolean Functions}

Smolensky [1] used the differential dimension of Boolean functions to lower-bound symmetriccircuit complexity. In this paper we used communication-complexity argurnents to simplify the proofs. We now show that similar communication-complexity arguments can be used to lower bound the differential dimension of Boolean functions

Let $\mathrm{S}$ be a finite set of points in the n-dimensional complex vector space $\mathrm{C}^{\mathrm{n}}$. Let $\mathrm{V}$ denote the space of functions from $\mathrm{S}$ to $\mathrm{C}$.

\section{Differential Dimension}

The differential dimension of an analytic function $g: C^{\prime \prime} \rightarrow C$ over $\mathrm{S}$ is the dimension of the subspace of $\mathrm{V}$ spanned by the restrictions to $\mathrm{S}$ of $\mathrm{g}$ and all of its partial derivatives.

Since we are concerned with functions that interpolate Boolean functions, we assume without loss of generality that $S=\{0,1\}^{\{1, \ldots, n\}}$.

\section{Differential Dimension of Boolean Functions}

The differential dimension of a Boolean function $\mathrm{f}:\{0,1\}^{\{1, \ldots, n\}} \rightarrow\{0,1\}$ is the minimal differential dimension over $S=\{0,1\}^{\{1, \ldots, n\}}$ of any analytic function $g: C^{\text {n }} \rightarrow C$ that interpolates $\mathrm{f}$.

Let $g: \mathrm{C}^{\mathrm{n}} \rightarrow \mathrm{C}$ be an analytic function and let $\mathrm{v} \in \mathrm{C}^{\mathrm{n}}$. The shifted function $g_{v}$ is defined by: $g_{v}(x)=g(x-v) \vee x \in C^{n}$.

Proposition 1 ([1]) The subspace of V spanned by all the partial derivates of all orders of $g$ restricted to $\mathrm{S}$ coincides with the subspace of $\mathrm{V}$ spanned by all the shifts of $g$ restricted to $\mathrm{S}$.

Thus if $g$ interpolates a given Boolean function $f$, then the dimension of the space spanned by the shifts $g$, for all $v \in S=\{0,1\}^{\{1, \ldots, n\}}$ lower bounds the differential dimension of $g$.

Any function $g$ restricted to the set $S=\{0,1\}^{\{1, \ldots, n\}}$ can be viewed as a $2 "$-dimensional vector in $\mathrm{C}^{\mathrm{n}}$; each coordinate of the vector is the value of $g$ at a distinct point in $\mathrm{S}$. For any $v_{i} \in \mathrm{S}$, we shall represent the shift $g_{v_{i}}$ restricted to $\mathrm{S}$ as a $2 "$-dimensional vector, and denote 
it as $g_{v_{i}, S}$. Then the dimension spanned by the shifts $g, g_{v_{2}}, \cdots, g_{v_{k}}, v_{i} \in S$, is the rank of the following $2^{\prime \prime} \mathrm{x} \mathrm{k}$ matrix:

$$
\left[g_{v_{1}, S} g_{v_{2}, S} \cdots g_{v_{k} s}\right]
$$

Lemma 6 The differential dimension of a Boolean function $f$ is $\Omega(r)$, where

$$
r=\max \left\{\operatorname{rank}\left(M_{f, X, Y}\right):\{X, Y) \text { partitions }\{1, \ldots, n\}\right\}
$$

Proof: Let $\{X, Y)$ partition $\{1, \ldots, n)$ and let $M_{f, X, Y}$ be the corresponding function matrix. Choose $\mathrm{k}=\operatorname{rank}\left(M_{f, X, Y}\right)$ linearly independent columns of $M_{f X Y}$, and let $\left\{y_{1}, y_{2}, \cdots, y_{k}\right\}$ be the set of $\mathrm{Y}$-inputs corresponding to the chosen columns. Let $g(x, y)$ interpolate $f$ and consider the following $k$ shifts: $g_{\left(0,-y_{1}\right)}, g_{\left(0,-y_{2}\right)}, \cdots, g_{\left(0,-y_{k}\right)}$. One can show the following for the shifts $g_{\left(0,-y_{i}\right)}$, restricted to $S=\{0,1\}^{\{1, \ldots, n\}}$ : 1) $g_{\left(0,-y_{i}\right)}(x, 0)=g\left(x, y_{i}\right)=f\left(x, y_{i}\right)$, is known for every $x \in\{0,1\}^{x}$ and the values of $g_{\left(0,-y_{i}\right)}(x, y)$ might be undetermined if $y \neq 0$; 2 ) If the entries of the vector $g_{\left(0,-y_{i}\right), S}$ are arranged so that the first $2^{|X|}$ entries correspond to $(x, 0) \in\{0,1\}^{\{1, \ldots, n\}}$, then in the following $2^{n} \mathrm{x}$ k matrix

$$
Y_{k}=\left[g_{\left(0,-y_{1}\right), S} g_{\left(0,-y_{2}\right), S} \cdots g_{\left(0,-y_{k}\right) S}\right]
$$

the sub-matrix defined by the first $2^{|X|}$ rows are the $\mathrm{k}$ linearly independent columns (corresponding to Y-inputs $\left(y_{1}, \cdots, y_{k}\right)$ chosen from $M_{f, X, Y}$. Thus rank $\left(Y_{k}\right)=\mathrm{k}$. Hence by Proposition 1 , the differential dimension of any function $g$ interpolating the Boolean function $f$ is $\Omega\left(\operatorname{rank}\left(M_{f, X, Y}\right)\right)$.

The above result implies, for example, that the differential dimensions of the $n$-variable EQ and COMP are $\Omega\left(2^{n / 2}\right)$.

\section{References}

[1] $R$. Smolnesky. On interpolation by analytical functions with special properties and some weak lower bounds on the size of circuits with symmetric gates. In Proc. of the $31 \mathrm{st}$ Annual Symposium on Foundations of Computer Science, pages 628-631, 1990.

[2] H. D. Groger and G. Turán. On linear decision trees computing boolean functions. In Proc. of the ICALP (J.L.Albert, B.Monien, M.R.Artalejo eds.), pages 707-718, 1991. 
[3] I. Wegener. The Complezity of Boolean Functions. New York: Wiley, 1987.

[4] M. Furst, J. B. Saxe, and M. Sipser. Parity circuits and the polynomial time hierarchy. In Proc. of the 22nd Annual Symposium on Foundations of Computer Science, pages 260-270, 1981.

[5] A. A. Razborov. Lower bounds for the size of circuits of bolunded depth with basis $\{A, \$)$. In Math. Notes of the Academy of Science of the USSR, 41:4, pages 333-338, 1987.

[6] R. Srnolensky. Algebraic methods in the theory of lower bounds for boolean circuit complexity. In Proc. of the 19th Annual ACM Symposium on Theory of Computing, pages 77-82, 1987.

[7] A. Hajnal, W. Mass, P. Pudlák, M. Szegedy, and G. Turán. Threshold circuits of bounded depth. In Proc. of the 28th Annual Symposium on Foundations of Computer Science, pages 99-110, 1987.

[8]J. Hromkovic. Linear Lower Bounds on unbounded fan-in Boolean Circuits. Information Processing Letters, 21:71-74, 1985.

[9] A.C. Yao. Some complexity questions related to distributive computing. In Proc. of the 11th Annual ACM Symposium on Theory of Computing, pages 209-213, 1979.

[10] A.V. Aho, J.D. Ullman, and M. Yanakakis. On notions of information transfer in VLSI circuits. In Proc. of the 15th Annual ACM Symposium on Theory of Computing, 1983.

[11] K. Melhorn and E.M. Schmidt. Las vegas is better than determinism in vlsi and distributed computing. In Proc. of the 14th Annual ACM Symposium on Theory of Computing, 1982.

[12] J. Spencer. Ten Lectures on the Probabilistic Method. SIAM, 1987.

[13] M. Goldmann, J. Håstad, and A. Razborov. Majority gates vs. general weighted threshold gates. In Proc. of the 7th Annual Conference on Structure in Complezity Theory, 1992. 